\title{
PHASE SEPARATION IN HUBBARD MODEL
}

\author{
J.H. SAMSON \\ Department of Physics, Loughborough University \\ Loughborough, Leics LE11 3TU, United Kingdom
}

The IIartree-Fock ground-state phase diagram of the one-dimensional IIubbard model is calculated in the $\mu-U$ plane, restricted to phases with no charge density modulation. This allows antiferromagnetism, saturated ferromagnetism, spiral spin density waves and a collinear structure with unit cell $\uparrow \uparrow \downarrow \downarrow$. The spiral phase is unstable against phase separation near quarter-, half- and three-quarter-fillin: For large $U$ this occurs at hole (or electron) doping of $\left(3 t / \pi^{2} U\right)^{1 / 3}$ from half-filling.

PACS numbers: 75.10.Lp, 75.25.+z

\section{Introduction}

Although the exact ground state of the one-dimensional one-band Hubbard model with nearest-neighbour hopping is well known [1], systematic Hartree-Fock (IIF) studies of the same model are still of value. They are correct in the limit of large degeneracy, they represent an effective Hamiltonian in functional integral formalism, and they provide a toy model for understanding the magnetic phases of two- and three-dimensional systems (such as cuprate superconductors and transition metal alloys). Such studies also raise suggestive connections with state-selection problems in frustrated Ileisen!)erg magnets.

The present author has obtained the HF ground state phase diagram of the one-dimensional nearest-neighbour IIubbard model [2]

$$
H=-t \sum_{i=1}^{N_{a}} \sum_{s=\uparrow}^{\downarrow}\left[c_{i s}^{\dagger} c_{(i+1) s}+c_{i s}^{\dagger} c_{(i-1) s}\right]+U \sum_{i=1}^{N_{a}} n_{i \uparrow} n_{i \downarrow}
$$

for arbitrary band filling $n(0 \leq n \leq 2)$. The calculation was restricted to phases without charge density modulation; subject to this restriction, the full $n-U$ phase diagram was obtained.

The work here extends these results to the grand canonical case, presenting the $\mu-U$ phase diagram and the asymptotic form of the phase boundaries. Full details of the theory are given in the above reference. 


\section{Hartree-Fock phase diagram}

The unrestricted IIF approximation minimises the expectation value of the Ilubbard IIamiltonian (1) in the space of Slater determinants. These states are ground states of a non-interacting many-electron system in a potential specified by variational parameters.

We restrict consideration to the uniform phases, where the only spatial dependence is in the local magnetisation directions, and to macroscopic phase separation. In the latter case two uniform phases are in equilibrium, separated by a domain wall. There are two families of uniform phases:

- SSDW (spiral spin density wave, $\uparrow \nearrow \rightarrow \searrow$ ) of continuously varying wave vector $Q$. This has limiting cases

$$
\begin{aligned}
& Q=0: \text { FM (saturated ferromagnetism, } \uparrow \uparrow \uparrow \uparrow) . \\
& Q=\pi: \text { AFM (antiferromagnetism, } \uparrow \downarrow \uparrow \downarrow) .
\end{aligned}
$$

- DSDW (double spin density wave, or two interpenetrating antiferromagnetic sublattices with Néel vectors canted at an angle $\theta, \uparrow \nearrow \downarrow \swarrow)$.

For each point $(n, U)$ the energy of each family is minimised with respect to the exchange splitting and the angle $(Q$ or 0$)$. The IIF energy $E_{\mathrm{HF}}(n, U)$ is the lower of the minima for SSDW and DSDW.

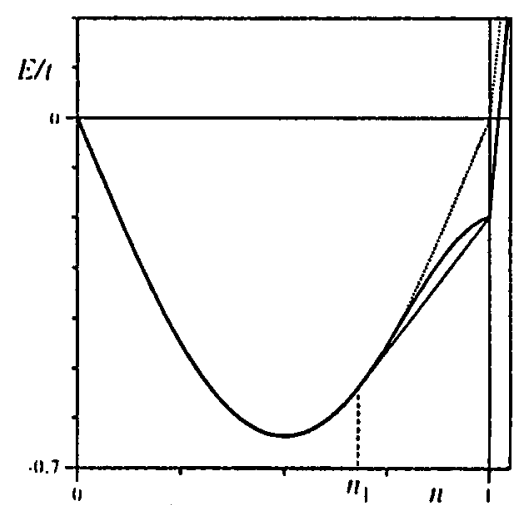

(a)

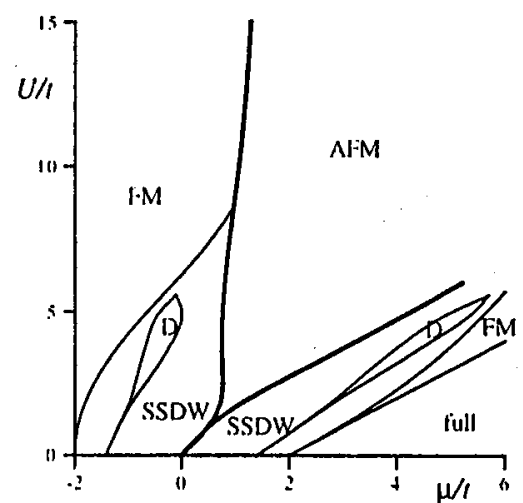

(b)

Fig. 1. (a) The HF energies for $U=10 t$. Bold curve: SSDW energy; dotted curve: FM energy; thin line: energy of phase-separated state. (b) The IIF phase diagram of the Hubbard model. The AFM phase has filling $n=1$, and the collinear DSDW phases (denoted "D") have filling $n=1 / 2,3 / 2$. The region marked "full" corresponds to filled bands $(n=2)$.

The condition for stability against macroscopic phase separation is that $E_{\mathrm{HF}}(n, U)$ be a convex function of $n$, or equivalently that the chemical potential

$$
\mu=\partial E_{\mathrm{HF}} / \partial n
$$


be an increasing function of $n$. If this does not hold, a Maxwell construction [3], shown in Fig. 1a, determines the fraction of each phase.

The figure shows the generic picture for $U>8.7 t$. For $n_{1}<n<1$ (and $1<n<2-n_{1}$ ) the HF ground state consists of a uniform AFM phase with $n=1$ in equilibrium with a hole-rich (electron-rich) uniform FM phase with $n=n_{1}\left(n=2-n_{1}\right)$. The chemical potential is pinned within the AFM gap and within the lower (upper) band of the FM phase. Additional carriers will simply move the domain wall. A similar effect is seen in supercell calculations of collinear configurations [4]. For $n<n_{1}$ (and $2-n_{1}<n$ ) the ground state is FM. Thus the SSDW is always unstable against separation into AFM and FM phases. (Note that the SSDW energy peels off from the FM energy, indicating $Q>0$, only above $n_{1}$.) The situation is slightly different for $U<8.7 t$ : near $n=1$ the SSDW is unstable against phase separation into an $A F M$ phase and a SSDW of longer wavelength.

The previous paper [2] presented the phase diagram in the $n-U$ plane. Figure $1 \mathrm{~b}$ shows the phase diagram in the $\mu-U$ plane. The areas shown here are pure phases, separated by second-order transitions (between FM and finite- $Q$ SSDW) and first-order transitions (between SSDW and collinear DSDW, between SSDW and AFM, and between FM and AFM). For large $U$, as a function of chemical potential there are two discontinuities in filling factor and in wave number. As $U$ is decreased, further discontinuities appear (although only the DSDW phase has been calculated).

\section{Form of phase boundaries}

We can compute the asymptotic form of the FM-AFM phase boundary for large $U$. By expanding the IIF energy of the AFM state at $n=1$ we find the gap to be

$$
\Delta=U-4 t^{2} / U+4 t^{4} / U^{3}+\ldots
$$

and the energy

$$
E_{\mathrm{HF}}(1, U)=-2 t^{2} / U+2 t^{4} / U^{3}+\ldots
$$

(This of course exceeds the Bethe Ansatz ground state energy $-4 \ln 2 t^{2} / U$ [5]). Drawing a tangent to the FM energy $-(2 t / \pi) \sin n \pi$ gives the form of the phase boundary for large $U$ as

$$
n_{1}=1-\left(\frac{3}{\pi^{2}} \frac{t}{U}\right)^{1 / 3}-\frac{t}{10 U}+\ldots
$$

or

$$
\mu=2 t-\left(\frac{3 \pi t}{U}\right)^{2 / 3} t+\ldots
$$

\section{Discussion}

The full HF solution to the one-dimensional Hubbard model is more difficult to compute, in general requiring a $4 N_{\mathrm{a}}$-dimensional minimisation. One might speculate on a possible devil's staircase for small $U$ : tongues, such as that for DSDW, would exist for all rational fillings. Similar behaviour is seen in the Falicov-Kimball 
model (where one spin state is immobile) [6]. For larger $U$ (in the Hubbard model) the tongues would disappear, thiming out the staircase. We would indeed expect the uniform SSDW phases to distort in such a way as to open a gap at the Fermi surface; the DSDW can be seen in such a way [7]. The phase separation seen here may appear in a microscopic form (as a soliton lattice) rather than the macroscopic form discussed here. Realistic terms, absent in the one-band Hubbard model, which suppress long-wavelength charge fluctuations, would tend to prevent macroscopic phase separation.

It is also interesting to note connections with the problem of state selection in frustrated IIeisenberg magnets. DSDW phases with varying $\theta$ are strictly degenerate in a classical Heisenberg model; here it is the itinerant nature of the magnetism which selects the collinear state.

We end by confessing that these results bear little relation to the true ground state of the one-dimensional one-band IIubbard model, which does not break symmetry in this way. However, they may provide a useful starting point for the IIubbard model in two and three dimensions and many-band Ilubbard models in one dimension.

\section{References}

[1] E.H. Lieb, F.Y. Wu, Phys. Rev. Lett. 20, 1445 (1968).

[2] J.H. Samson, J. Phys., Condens. Maller 8, 569 (1996).

[3] M. Marder, N. Papanicolaou, G.C. Psatalkis, Phys. Rev. B 41, 6920 (1990).

[4] A.N. Andriotis, E.N. Economou, C.M. Soukoulis, J. Phys., Condens. Maller 5, 4505 (1993).

[5] See e.g. P. Fulde, Electron Correlations in Metals and Solids, Springer, Berlin 1991, p. 290.

[6] J. Lach, R. Łyżwa, J. Jędrzejewski, Phys. Rev. B 48, 10783 (1993).

[7] Y. Suzumura, N. Tanemura, J. Plyys. Soc. Japan 64, 2298 (1995); N. Tanemura, Y. Suzumura, J. Plyss. Soc. Japan 66, 1792 (1996). 